\title{
Robust Die Compensation in Sheet Metal Design through the Integration of Dual Response Surface and Shape Function Optimization
}

\author{
Michele Bici $\mathbb{D}^{1},{ }^{1}$ Francesca Campana $\mathbb{D D}^{1},{ }^{1}$ Flavio Cimolin, ${ }^{2}$ and Leopoldo Rizzo ${ }^{3}$ \\ ${ }^{1}$ Dipartimento di Ingegneria Meccanica e Aerospaziale, Università di Roma "La Sapienza," Via Eudossiana 18, 00184 Roma, Italy \\ ${ }^{2}$ AMET Italy S.R.L., Via Livorno 60, 10144 Torino, Italy \\ ${ }^{3}$ Centro Sviluppo Materiali S.p.A., Via di Castel Romano 100, 00128 Roma, Italy \\ Correspondence should be addressed to Michele Bici; michele.bici@uniromal.it
}

Received 30 November 2018; Revised 15 March 2019; Accepted 14 April 2019; Published 23 May 2019

Academic Editor: Paolo Manfredi

Copyright (C) 2019 Michele Bici et al. This is an open access article distributed under the Creative Commons Attribution License, which permits unrestricted use, distribution, and reproduction in any medium, provided the original work is properly cited.

\begin{abstract}
In sheet metal forming, springback represents a major drawback increasing die set-up problems, especially for ultra-high strength steels. Finite Element Analysis is a well-established method to simulate the process during design, and multicriteria optimizations, for example, via surrogate models, are investigated in order to develop integrated design. Since to take into account also springback compensation die design may involve a large number of geometric variables, this paper presents a robust design formulation, based on the adoption of the shape function optimization, to describe springback in terms of weights directly associated to global shape variations of the die shape. Doing so, multicriteria optimization, which involves also die compensation, can be set up in a more intuitive approach, as requested in the preliminary steps of die design. After the introduction of the industrial problem, the mathematical formulation of the shape function optimization is presented together with its novel extension to Robust Design, which is based on the Dual Response Surface. Through a test case derived from the head part of a B-pillar, stamped from a Dual Phase sheet $1.5 \mathrm{~mm}$ thick, this novel extension investigates the effect of $6 \%$ variation from nominal values of initial yield stress and thickness. Results demonstrate the feasibility of the procedure, underlying that an optimal compensation may not be optimal in terms of process robustness.
\end{abstract}

\section{Introduction}

In sheet metal forming, springback is due to the elastic recovery of the stamping forces after tool removal. It gives global shape deviations (opening of bendings, deflections, ...) if no geometric constraints may contrast it. Generally speaking, initial thickness, component shape, stamping forces, and material characteristics are major responsible of its severity.

Springback prevention and optimization represent an important goal for the integrated design of sheet metal formed parts, since shape distortion of components may obstacle correct assembly. In particular, because of their enhanced mechanical properties, Advanced High Strength Steels (AHSS) are often applied to reduce weight of structural components by means of thickness reduction, but they present a more pronounced springback due to an increased stamping force [1]. As a consequence, the interest about numerical optimization of springback has increased and Finite Element Analysis (FEA) plays an important role to reduce die try-out efforts, similarly to what already happens for other stamping problems [2-6].

The industrial approach to reduce springback consists in die compensation, which is a shape modification of the die, able to overbend or overstretch critical areas of the component. Therefore, numerical optimization of springback mainly consists of finding the optimal die shape able to compensate component distortion after springback. Many researches are concerned with this topic and solve the problem with different approaches.

The Spring-Forward method (SF) represents one of the first formulations developed in order to numerically reduce springback by die compensation [7]. It works on the 
equilibrium of the forces that act on the component at the end of stamping. In this phase, the tools apply to the component a stress field that induces springback as soon as they are removed. An elastic simulation on the free part, loaded according to this stress field with reversed sign, may give a final displacement that is assumed as a die compensation of the applied load.

An alternative method is the Smooth Displacement Adjustment (SDA) or its first formulation, simply called Displacement Adjustment (DA) [8]. The basic idea of the DA method is to adjust the die surface in the opposite direction of the springback deviation (DA in a single stage). To achieve improved results, this approach is usually applied iteratively, since tool geometry converges to a shape able to reproduce the optimal displacement field (the target part) by leading to zero the displacement deviation. In [9], a comparison between DA and SF is discussed according to the analytical bar stretch bending model, pointing out the supremacy of the DA method, as discussed also in [10]. Unfortunately, in the DA method, the compensation field is generally applied to the mesh nodes and this may give unacceptable tools or rough solutions in case of complex shapes [11]. To solve this problem, the Smooth DA method (SDA), or the Surface Controlled Overbending method (SCO), has been proposed [12] and successfully applied also to industrial case studies [13]. The SDA applies the DA formulation on an analytical approximation of the discrete mesh that is made according to polynomial basis-functions of $\mathrm{x}, \mathrm{y}, \mathrm{z}$, while, in the SCO, the shape modifications are applied to the control points of NURBS surfaces that represent the CAD models of the component and the die [14]. A more recent approach to springback compensation is discussed and applied in $[15,16]$. It approaches the die compensation as an inverse problem solved by shape functions optimization. Shape functions are derived from modal analysis of the final component and modifications of the die are obtained by combining similar shape functions that are related to the die. The linear relationship, among die shape functions and final component's shape functions, allows to describe the problem of springback in terms of a more limited number of variables, which is suitable to be solved in an optimization fashion.

In this paper, the die compensation by shape function optimization is integrated into Robust Design, to demonstrate its ability in support sheet metal forming design optimization thanks to a reduction of the design space related to the geometrical shape variables. Generally speaking, Robust Design techniques are particularly interesting to reduce product quality variations induced by random process variables (e.g., blank properties within their tolerance range). Many test cases and applications related to the robustness of forming quality are present in literature [17-21]. The specific methodologies applied in this field consist of different statistical techniques such as Dual Response Surface modeling or Monte Carlo method coupled with numerical Design of Experiments (DOE). In [22-26], some examples that involve springback are discussed. They investigate springback sensitivity to noise variables such as lubrication or material characteristics. In particular, in [27], the interaction between tool shape and blank yield stress variation is found as the most significant interaction in comparison with other process variables such as, for example, the blankholder force. In [28], a general overview of the optimization problems in sheet metal forming design is shown from the point of view of surrogate modeling, also known as meta-modeling. Critical issues for widespread applications of these numerical tools in integrated design are the following:

(i) The difficulty of defining proper objective functions

(ii) The large number of variables from different technical aspects

(iii) The complexity of the interactions among them due to the nonlinearity of the process (large strains and plasticity)

Practical experience in the press-shop usually helps decisions related to these issues and their introduction in FEA optimization loops has been made with different levels of complexity: from single objective function definition up to knowledge based design [17, 29, 30].

In [31], a compensation strategy, suitable to include also process robustness, is claimed as necessary from the industrial point of view. Nevertheless, optimization processes with die compensation are not so frequent due to the difficulty of obtaining shape continuity and avoiding time-consuming loops between FEA and CAD model since they may include a large number of geometric variables. In [32], an evolutionary algorithm, based on neural network, is applied to springback to demonstrate the efficacy and efficiency of parametric optimization in sheet metal design.

After a brief introduction of the theoretical background of the adopted die compensation strategy presented in Section 2, Section 3 explains the Robust Design technique known as Dual Response Surface (Section 3.1) and its integration with the adopted die compensation strategy for robust design (Section 3.2). In Section 4, the test case related to the head part of a B-pillar is described and the results of its die compensation are shown and discussed in Section 5, and then Section 6 shows the results of the proposed integration with the Dual Response Surface and the related discussion is in Section 7. Finally, the main conclusions are outlined in Section 8.

\section{Die Compensation Strategy}

The mathematical formulation of the problem has been firstly presented in [15]. In FEA, both die and deep drawn sheet can be described by a mesh, represented, respectively, by the vectors $\mathbf{s}_{\text {nom }} \in \mathfrak{R}^{N}$ and $\mathbf{u}_{\text {nom }} \in \mathfrak{R}^{M}$. In principle, we should think of $\mathbf{u}_{\text {nom }}$ as the mesh associated to the desired component configuration as conceived by the designer, while $\mathbf{s}_{\text {nom }}$ is usually computed by translation and offset of the component geometry. By means of FEA, it is possible to predict the final configuration of the formed sheet as a function of the die shape. Let us denote by $G$ the function computed by the FE code that, starting from a certain die configuration $s$, gives as output the final configuration of the sheet $\mathbf{u}$. Usually, because of springback, it can be verified that $G\left(\mathbf{s}_{\text {nom }}\right) \neq \mathbf{u}_{\text {nom }}$, where the subscript "nom" stands for the nominal configuration defined by the designer. 
The springback compensation problem can then be expressed as the problem of seeking that peculiar die configuration $\mathbf{s} *$ that minimizes the objective function

$$
e(\mathbf{s})=\left\|\mathbf{u}_{\text {nom }}-G(\mathbf{s})\right\|
$$

where the norm is a suitable choice, for example, the Euclidean one.

The best possible choice would be to find out a die configuration $\mathbf{s} *$ such that Equation (1) is found equal to zero, but this is, in general, neither mathematically nor technologically assured to exist, because of the high nonlinearity of the function $G$ and of the possibility of undercuts, respectively. Considering that, usually, the $N$ nodal unknowns are of the order of 10.000, the computation of $G$ can be carried out through a very expensive FEA.

This means that it is fundamental to rewrite the optimization problem (1) in a form that depends on far less than $N$ variables in order to keep limited the total number of evaluations. The key idea of the Shape Function approach is to define a reduced number of basic shapes that, assembled together by linear combination, can be used to well approximate the configurations of both $\mathbf{s}$ and $\mathbf{u}$. For a generic nodal configuration $\mathrm{x}$, one can define an ordered succession of eigenvalue-eigenvector pairs $\left\{\omega_{i}^{2}, \Psi_{i}\right\}$ associated to the eigenvalue problem:

$$
\mathbf{K} \mathbf{x}=\omega^{2} \mathbf{M} \mathbf{x}
$$

$\mathbf{K}$ and $\mathbf{M}$ are the stiffness and mass matrices, respectively. These correspond to the well-known vibration modes, employed in many engineering applications associated to structural analysis [33].

$\mathbf{K}$ and $\mathbf{M}$ are orthogonal among each other since

$$
\begin{gathered}
\Psi_{\mathrm{i}}^{\mathrm{T}} \mathbf{M} \Psi_{\mathrm{j}}=\delta_{\mathrm{ij}} \\
\Psi_{\mathrm{i}}^{\mathrm{T}} \mathbf{K} \Psi_{\mathrm{j}}=\delta_{\mathrm{ij}},
\end{gathered}
$$

where $\Psi_{\mathrm{i}}$ stands for the ith shape function and $\delta_{\mathrm{ij}}$ is equal to 1 if $i=j$; otherwise, it is null.

Setting $\mathbf{M}=\mathbf{I}$, where $\mathbf{I}$ is the identity matrix, $\boldsymbol{\Psi}_{\mathrm{i}}^{\mathrm{T}} \boldsymbol{\Psi}_{\mathrm{j}}=\delta_{\mathrm{ij}}$, so they are orthogonal functions. This property makes the shape functions a very suitable choice for the representation of the deformations of both $\mathbf{s}$ and $\mathbf{u}$ around their nominal configurations, as explained in the following.

Let us then compute the sequence of shape functions $\mathbf{w}_{i}^{\mathrm{s}}$ and $\mathbf{w}_{i}^{\mathrm{u}}$, evaluated in the neighborhood of the nominal configuration of the die and the final sheet, respectively. A reduction of the problem size can be obtained by selecting only $n$ and $m$ shape functions for each of the two spaces. Doing so, a deformed die can be obtained through a linear combination of the shape functions on the die, weighted by the coefficients $\alpha_{i}$ :

$$
\mathbf{s}=\mathbf{s}_{\text {nom }}+\sum_{i=1}^{n} \alpha_{i} \mathbf{w}_{i}^{s}
$$

The final configuration of the sheet can be formulated according to

$$
\mathbf{u}=G\left(\mathbf{s}_{\text {nom }}+\sum_{i=1}^{n} \alpha_{i} \mathbf{w}_{i}{ }^{s}\right)=\mathbf{u}_{\text {nom }}+\sum_{j=1}^{m} \beta_{j} \mathbf{w}_{j}{ }^{u}+\mathbf{r}_{m}{ }^{u}
$$

where the last expression represents the resulting configuration in terms of the shape functions $\mathbf{w}_{j}^{u}$, collecting the reminder into the term $\mathbf{r}_{m}^{u}$. In this way, we have defined a map between the coefficients $\alpha_{i}$ associated to the configuration of the die and the coefficients $\beta_{j}$ related to corresponding stamped sheet.

Thanks to the orthogonality of the shape functions, coefficients $\beta_{j}$ are computed by

$$
\beta_{j}=\left(\mathbf{u}-\mathbf{u}_{n o m}\right) \cdot \mathbf{w}_{j}^{u}
$$

By changing the values of the coefficients $\alpha_{i}$, we can generate different die shapes, which, at the end of the simulation, will provide different pieces, which are read in terms of the coefficients $\beta_{j}$. Of course, the nominal die configuration corresponds to $\alpha_{i}=0$ for all $i$, while the nominal sheet configuration corresponds to $\beta_{j}=0$ for all $j$. Collecting the coefficients $\alpha_{i}$ and $\beta_{j}$ into the vectors $\boldsymbol{\alpha} \in \mathfrak{R}^{n}$ and $\boldsymbol{\beta} \epsilon$ $\mathfrak{R}^{m}$, it is possible to reformulate the problem of springback compensation in terms of these new variables. Doing so, with respect to the new bases, we obtain a problem that mimics the behavior of the original one. In analogy to the case, which leads from $\mathbf{s}$ to $\mathbf{u}$ through the function $G$, let us call $g$ the function that gives $\boldsymbol{\beta}$ from $\boldsymbol{\alpha}$. The optimization problem expressed in the shape function space is now to look for the die configuration $\alpha *$ that minimizes the objective function:

$$
\mathcal{E}(\boldsymbol{\alpha})=\frac{1}{2}\|g(\boldsymbol{\alpha})\|=\frac{1}{2} \boldsymbol{\beta}^{T} \boldsymbol{\beta}=\frac{1}{2} \sum_{j=1}^{m} \beta_{j}^{2}
$$

The gain in switching from optimization problem (1) to (7) is that we have reduced the number of unknowns from $N$ to $m$ through Equations (5) and (7), and the latter can be kept around 10-50 without losing too much accuracy in the reproduction of the deformations. This fact depends on the high suitability of the shape functions to reproduce springback-associated deformations, which span over the whole piece and rarely have localized influences on it; thus high frequency modes are less significant to describe springback displacement.

Solution of Equation (7) can be found through least squares optimization. A preliminary implementation applies the modified Gauss-Newton method, further improved by means of a line-search and through the introduction of a reduced basis technique for the advancing step estimation [15].

\section{Robust Design Formulation}

3.1. Theoretical Background. Robust Design (RD) is concerned with minimizing the random effects of one or more noise variables on the expected quality of a product. In 
manufacturing processes, such as sheet metal forming, blank characteristics may be seen as noise variables that randomly change within their tolerance range, producing a scatter around the target value of a quality response that can be associated to springback or other structural stamping defects.

In order of time, Taguchi's method represents the first methodical approach to RD, while meta-modelling and Dual Response Surface have been introduced next, to overcome some theoretical problems present in Taguchi's approach $[35,36]$. Nevertheless, all of them are based on Design Of Experiments (DOE) techniques [37]. They make a global investigation of the design space, finding main and interaction effects of variables on the quality response, in a less expensive way. A RD problem divides the design space in control and noise factors. The experiments, which are performed according to noise factors, permit to evaluate response scattering; meanwhile a proper variation of control factors, able to interact with noise, permits to find out both optimal conditions that move the response mean to the target and the response variance to a minimum.

In the most recent years, the decrease of computational costs of FEA has spread the adoption of meta-modelling techniques substituting Taguchi's simplified approach with the Dual Response Surface or other stochastic methods [38]. The main objective of meta-models is to describe the relationships between design parameters and quality response analytically. Taguchi's analysis is often limited to main and linear effects while the Dual Response Surface, thanks to the adoption of a central composite design, is suitable also for nonlinear investigations. This method describes both mean and variance analytically, starting from a general meta-model of the response (mixed model approach).

Assuming $k$ experiments, made according to a central composite design that joins together control and noise factors, the $i$ th response, $\gamma_{i}$, can be written as

$$
\begin{array}{r}
y_{i}=\gamma_{0}+f^{T}\left(\bar{c}_{i}\right) \bar{\gamma}+\bar{n}_{i}^{T} \bar{\delta}+f^{T}\left(\bar{c}_{i}\right) \Lambda \bar{n}_{i}+\bar{n}_{i}^{T} \Theta \bar{n}_{i}+\varepsilon_{i} \\
i=1, k
\end{array}
$$

where the following are considered:

(i) $\gamma_{0}$ stands for the constant value of the meta-model (equals the total mean of the experimental responses)

(ii) $f^{T}\left(\bar{c}_{i}\right)$ represents the transpose vector of the linear and nonlinear terms (quadratic and interactions) of the control factor vector associated to the $i$ th run, $\bar{c}_{i}$

(iii) $\bar{\gamma}$ stands for the unknown coefficient vector of the control variable terms of the meta-model

(iv) $\bar{n}_{i}$ stands for linear terms of the noise factor vector at the $i$ th run

(v) $\bar{\delta}$ stands for the unknown coefficient vector of the noise variable terms of the meta-model

(vi) $\Lambda$ and $\Theta$ are the unknown coefficients to describe, respectively, the effect of noise variables in terms of interaction with control factors and interaction with themselves (vii) finally, $\varepsilon_{i}$ represents the error of the meta-model when the $i$ th response is evaluated

From Equation (8), mean and variance of the response are computed under the hypothesis of $\varepsilon_{i}$ independent with zero mean:

$$
E\left(y_{i}\right)=\gamma_{0}+f^{T}\left(\bar{c}_{i}\right) \bar{\gamma}+\operatorname{tr}(\Theta \Lambda)
$$

and

$$
\begin{aligned}
\operatorname{var}\left(y_{i}\right)= & {\left[\bar{\delta}+\Lambda^{T} f\left(\bar{c}_{i}\right)\right]^{T} \Phi\left[\bar{\delta}+\Lambda^{T} f\left(\bar{c}_{i}\right)\right] } \\
& +2 \operatorname{tr}\left\{(\Theta \Phi)^{2}\right\}+\sigma_{\varepsilon}^{2}
\end{aligned}
$$

Usually, quadratic terms of noise factors are not investigated, so $\Theta$ is null together with the trace computation of Equations (9) and (10). $\Phi$ represents the noise variance-covariance matrix. It is diagonal and it can be evaluated by a statistical knowledge of the investigated process (e.g., $\Phi_{u}=\sigma^{2}=1 / 9$ if $99.7 \%$ of the materials are in a $6 \sigma$ tolerance field and the noise levels of the DOE change from the coded values -1 and 1 ).

From these considerations we derive that the mean value of the response depends only on the control variables while the variance of the response is due to the noise factors both in terms of their linear coefficients $(\delta)$ and of their interactions with the control variables $(\Lambda)$. A regression algorithm able to find $\gamma_{0}, \bar{\gamma}, \bar{\delta}$, and $\Lambda$ allows to evaluate Equations (9) and (10) finding the relevance of each term of the meta-model and, so, the effects of the noise factors on the variance of the response.

3.2. Proposed Formulation for Robust Die Compensation. To explore how an optimal compensated die may reduce noise effects due to the tolerance of process variables, small perturbations of the die shape must be analyzed. The problem is solved looking for a set of perturbations able to minimize response scattering due to noise. According to Equation (1), we can say that in such configuration a small noise perturbation of the blank characteristics may change the norm according to a linear superposition of the effects:

$$
\begin{aligned}
e(\mathbf{s} *+\Delta \mathbf{s}) & =\left\|\mathbf{u}_{n o m}+\Delta \mathbf{u}-G(\mathbf{s} *+\Delta s)\right\| \\
& =\|\Delta \mathbf{u}-G(\Delta \mathbf{s})\| .
\end{aligned}
$$

In these terms, the robust design approach is looking for optimal compensated die through the search of a $\Delta \mathbf{s}$ able to minimize $e$.

Generally speaking, assuming $k$ shapes of deformation able to compensate noise effects, the problem defined in Equation (11) becomes

$$
e\left(\Delta a_{j}\right)=\left\|\Delta u-G\left(\sum_{j=1}^{k} \Delta a_{j} \mathbf{w}_{j}^{s}\right)\right\|
$$

Assuming as control factors a set of perturbations of the die modal weights, $\Delta \alpha_{j}$, common robust design strategies (e.g., Dual Response Surface) may be applied by deriving a set of modified tools according to a DOE as shown in the example of Figure 1. 


\begin{tabular}{|c|c|c|c|}
\cline { 2 - 3 } \multicolumn{1}{c|}{} & $\Delta \boldsymbol{\alpha}_{1}$ & $\boldsymbol{\Delta} \boldsymbol{\alpha}_{2}$ & \multicolumn{1}{c}{} \\
\hline tool shape \#1 & -1 & +1 & $=s *+\left(\Delta \alpha_{1} \boldsymbol{W}_{1}+\Delta \alpha_{2} \boldsymbol{W}_{2}\right)$ \\
\hline tool shape \#2 & -1 & -1 & $=s *+\left(\Delta \alpha_{1} \boldsymbol{W}_{1}+\Delta \alpha_{2} \boldsymbol{W}_{2}\right)$ \\
\hline tool shape \#3 & +1 & +1 & $=s *+\left(\Delta \alpha_{1} \boldsymbol{W}_{1}+\Delta \alpha_{2} \boldsymbol{W}_{2}\right)$ \\
\hline tool shape \#4 & +1 & -1 & $=s *+\left(\Delta \alpha_{1} \boldsymbol{W}_{1}+\Delta \alpha_{2} \boldsymbol{W}_{2}\right)$ \\
\hline
\end{tabular}

FIGURE 1: DOE scheme for die modification according to the proposed approach.

In this example, the experimental plan analyzes control factors that represent two global die modifications according to the modal shapes $\boldsymbol{w}_{1}$ and $\boldsymbol{w}_{2}$. They are applied on the optimal compensated shape, $s *$, by a linear combination of their die modal weights $\Delta \alpha_{1}, \Delta \alpha_{2}$ that change according to a 2-level full-factorial design (coded levels $[-1,+1]$ ). Doing so, die geometrical continuity is guaranteed by the smoothness of shape functions that may be affected only by the quality of the meshes adopted in the FEA.

According to this example, the general workflow of the procedure is described by the following steps:

(1) Build the compensated configuration of the tools (target configuration) through the application of the Shape Function optimization.

(2) Define the set of modal shapes as control factors and perturb their weights according to a DOE (e.g., central composite design for the Dual Response Surface or crossed arrays for Taguchi's method).

(3) Iteratively, for each run of the DOE,

(a) build other tools (blankholder and punch) by offset and translation on the die elements of the mesh

(b) check undercuts

(c) perform the simulation of the forming process

(d) coarsen the mesh of the formed part

(e) put constraints on the part and perform springback simulation

(f) remesh the part in order to obtain a standard mesh for all runs

(g) align the part to an absolute reference frame

(h) compute springback quality response able to evaluate current shape distortion

(4) Finally, analyze the collected springback quality responses to find the optimal solution according to one of the Robust Design procedures.

If the Dual Response Surface is applied, a meta-model for the mean and the variance of the process has to be fit according to Equations from (8) to (10), and, then, the optimal point may be found by a constrained minimization technique of the MSE.

The general workflow of the procedure requires only two major precautions: one related to the verification of possible

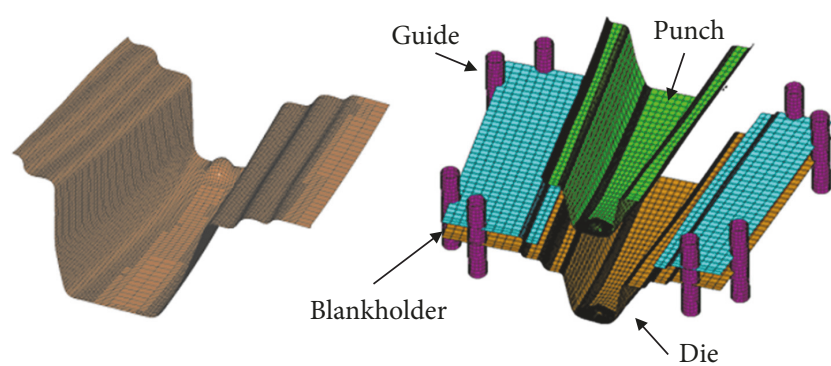

FIgURE 2: Test case: FEA nominal shape (on the left); FE model of the tools (on the right).

undercuts and the other to the adoption of a proper quality response that may be able to evaluate springback distortion. Concerning the adoption of a proper springback quality response, it is important to guarantee the significance of the results. Although many formulations can be correlated to FEA results [29], usually in die design and experimental validation it is based on the final displacement after springback. Depending on the complexity of the shape, it can be defined by monitoring a set of relevant points (the most affected by springback) or by monitoring the overall set of nodes. In the latter case, final springback is computed as an averaged value weighted with the element area of the mesh.

\section{Test Case Description and FEA Set-Up}

The proposed approach is here applied on a case study derived from the head part of a B-pillar (Figure 2, on the left), stamped from a Dual Phase (nominal yield stress equal to $1100 \mathrm{MPa}$ ) blank $1.5 \mathrm{~mm}$ thick. The general workflow for robust die compensation has been implemented through TCL scripts in Hyperworks, adopting as FEA code LS-Dyna.

FEA set-up involves two steps: the first one is related to the deep drawing simulation and the second one to the springback solution. The first one has been made adopting the explicit solver LS-DYNA according to the model shown in Figure 2, on the right. The second one switched shell formulation and the solver to the implicit solution.

In the deep drawing, all the components have been meshed by rigid shell elements, except the blank that represents the only deformable part. During the computation, its mesh is automatically refined where necessary (initial number of elements 960; final number after remeshing 13473). The blankholder force has been evaluated by splitting the total force that is currently applied to stamp the B-pillar, according to a proportional value of the effective area of the case study (final value: $350 \mathrm{kN}$ ). The punch load has been applied through a prescribed rigid motion according to stamping simulation practice. According to $[5,6,39]$ and considering that the adopted material is an AHSS, the plastic behavior has been modeled by Chaboche's formulation [34], a combined isotropic-kinematic hardening model with six parameters, reported in Table 1.

At the end of the deep drawing simulation, the tools are removed and the final springback solution is obtained by the implicit-solver computation. This simulation is made on a 
TABLE 1: Material parameters of the adopted combined isotropic-kinematic hardening model [34].

\begin{tabular}{lccccc}
\hline $\operatorname{Hard1}(\mathrm{GPa})$ & $\beta$ & $H a r d K 1(\mathrm{GPa})$ & $\gamma_{1}$ & HardK2 $(\mathrm{GPa})$ & $\gamma_{2}$ \\
\hline-60.000 & 150.0 & 1.200 & 5.00 & 88.000 & 150.00 \\
\hline
\end{tabular}

TABLE 2: Die compensation by the shape function optimization: target configuration in terms of die modal weights.

\begin{tabular}{lccccc}
\hline$\alpha_{1}$ & $\alpha_{4}$ & $\alpha_{5}$ & $\alpha_{7}$ & $\alpha_{8}$ & $\alpha_{9}$ \\
\hline-559.63 & 12.18 & 16.02 & -82.21 & -57.6 & 21.49 \\
\hline
\end{tabular}

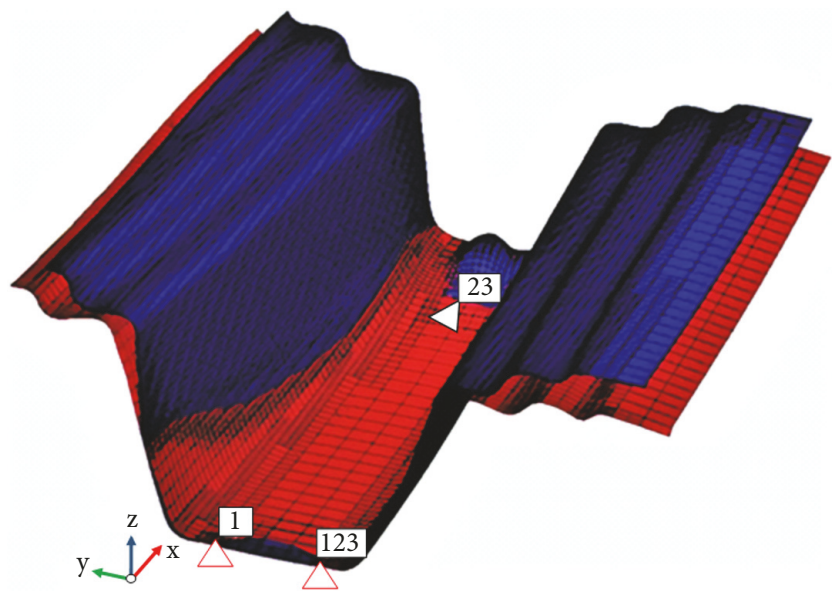

FIGURE 3: Nominal configuration before springback (blue mesh) and after springback (red mesh). Triangles represent the 3 nodes with the translational DOFs locked in the springback solution.

coarser mesh that has been assumed as the nodal reference springback solution for the computation of the springback quality response. It is an average value, which is weighted on the element areas, of the nodal difference between current and reference springback solution, after model repositioning on an absolute reference frame.

Then, the quality response is computed as an average of nodal values, weighted on the element area. Figure 3 shows the nominal shape (the blue one) compared with its final springback (the red one), which is going to be the target solution for the die compensation described in the next section.

Figure 3 also shows the symbols associated to the nodal constraints applied to prevent rigid body motions (red triangles). They consist of a set of zero displacement conditions in $\mathrm{x}, \mathrm{y}$, and $\mathrm{z}$ directions on three nodes at the bottom of the component. The same three nodes were also adopted to define the absolute reference frame for the repositioning of the modified sheet configurations on its nominal one.

\section{Die Compensation: Results and Discussion}

According to the formulation set in Section 2, only 6 shape functions bring variations of the global shape, effectively. Table 2 shows their values to compensate springback, while Figure 4 shows a comparison of the final shape obtained by die compensation (cyan surface), nominal shape (blue

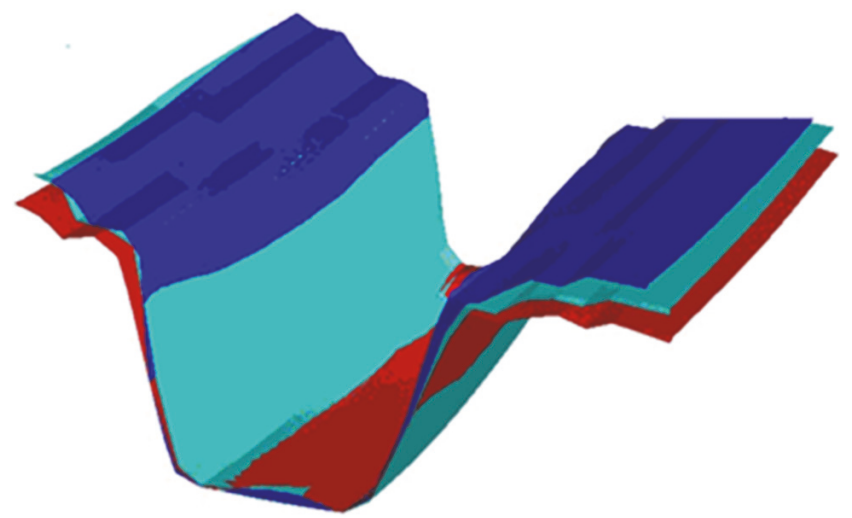

FIGURE 4: Springback solution after die compensation: compensated shape (cyan surface), original springback shape (red surface), and nominal shape (blue surface).

surface), and original, uncompensated, springback shape (red surface).

In terms of springback quality response, the compensation algorithm is able to reduce springback of $45 \%$, with a springback reduction at the most critical flange (on the frontleft of Figure 4) from an initial displacement of $20.1 \mathrm{~mm}$ to a final one of $11.3 \mathrm{~mm}$. Although the result could appear quite distant from a complete compensation, actually it is very close to the optimum, since there is a very sharp undercut constraint, which prevents the algorithm to proceed further in the direction of springback reduction.

Considering the obtained compensated shape from the shape functions point of view, only three shape functions $\left(\alpha_{1}\right.$, $\left.\alpha_{7}, \alpha_{8}\right)$ are the most effective on the shape variation from the target to the compensated solution. It can be revealed comparing among them the magnitude of the die modal weights. Figure 5 shows the associated modes, giving evidence of how they concur to the final distortion after springback. In particular, all of them affect the flange distortion, the first mode and the eighth on the bottom of the shape, and the seventh and the eighth mainly on the vertical areas.

Figure 6 shows an experimental comparison between the compensated part and the nominal geometry (in this case, the comparison has been focused outside the flange areas, considering that they must be cut before the assembly). Inside the figure, at the vertical wall, the correction leads to a maximum value of $-1.55 \mathrm{~mm}$ from the original springback distance of $10.28 \mathrm{~mm}$. 
TABLE 3: DOE range of the modal weights assumed as control factors.

\begin{tabular}{lccc}
\hline & $\alpha_{1}$ & $\alpha_{7}$ & $\alpha_{8}$ \\
\hline Lower bound & -634.63 & -157.21 & -132.60 \\
Upper bound & -484.63 & -7.21 & 0 \\
\hline
\end{tabular}

TABLE 4: Coded levels adopted for control and noise variables according to the central composite design.

\begin{tabular}{lccccc}
\hline Coded level & $\alpha_{1}$ & $\alpha_{7}$ & $\alpha_{8}$ & $\sigma_{y}(\mathrm{GPa})$ & 1.034 \\
\hline$-s$ & -634.63 & -157.21 & -132.598 & 1.072 & 1.410 \\
-1 & -591.16 & -113.74 & -89.131 & 1.100 \\
0 & -559.63 & -82.21 & -57.598 & 1.128 & 1.500 \\
1 & -528.09 & -50.67 & -26.064 & 1.162 & 1.538 \\
$S$ & -484.63 & -7.21 & 0.000 & 1.590 \\
\hline
\end{tabular}

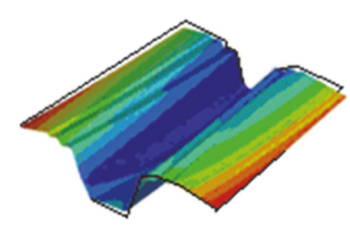

$\alpha_{1}=$ Shape 1

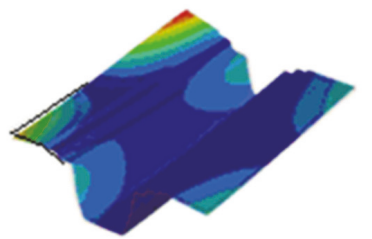

$\alpha_{7}=$ Shape 7

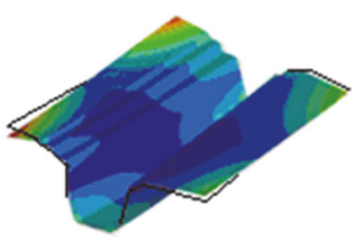

$\alpha_{8}=$ Shape 8

FIGURE 5: Modal shapes associated to the three most effective modal weights.

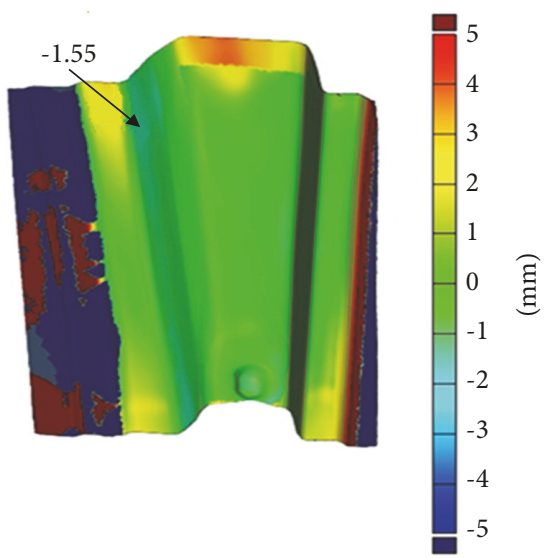

FIGURE 6: Experimental comparison between compensated shape and nominal shape.

\section{Robust Design Application: Results}

Starting from the compensation found in the previous section, the interactions between die shape and blank's scattering have been studied, according to the Dual Response Surface strategy explained in Section 3.2.

Considering the relevance of $\left(\alpha_{1}, \alpha_{7}, \alpha_{8}\right)$, they have been assumed as control variables of the problem, leaving the others fixed at the target values. Table 3 summarizes their ranges, considered during the robust design optimization.

They were defined not so wide to preserve the preliminary die compensation that may be seen as the first optimization step to set the average behavior of the process to the target.
The investigated noise factors are concerned with blank scattering: the initial yield stress, $\sigma_{y}$ and the initial blank thickness, $t$. Their variability has been set to $6 \%$ of the target conditions, which are $1100 \mathrm{MPa}$ and $1.5 \mathrm{~mm}$, respectively.

Table 4 shows the values assigned in the DOE according to the five coded values applied as requested in a central composite design. Table 5 shows the central composite design together with the springback quality response, $Q$, computed at each run.

The meta-model derived from these runs is represented by

$$
\begin{aligned}
Q= & \gamma_{0}+\gamma_{1} \alpha_{1}^{2}+\gamma_{2} \alpha_{1}+\gamma_{3} \alpha_{7}^{2}+\gamma_{4} \alpha_{7}+\gamma_{5} \alpha_{8}^{2}+\gamma_{6} \alpha_{8} \\
& +\gamma_{7} \alpha_{1} \alpha_{7}+\gamma_{8} \alpha_{1} \alpha_{8}+\gamma_{9} \alpha_{7} \alpha_{8}+\delta_{1} \sigma_{y}+\delta_{2} t \\
& +\Lambda_{11} \sigma_{y} \alpha_{1}+\Lambda_{12} \sigma_{y} \alpha_{7}+\Lambda_{13} \sigma_{y} \alpha_{8}+\Lambda_{21} t \alpha_{1} \\
& +\Lambda_{22} t \alpha_{7}+\Lambda_{23} t \alpha_{8}
\end{aligned}
$$

It includes quadratic terms with interactions for the control variables and linear terms for the noise factors together with their interactions with the control variables.

Table 6 shows the regression coefficients of the metamodel.

Table 6 shows that quadratic terms of the control factors are relevant; $\sigma_{y}$ is the most significant noise variable while $t$, in the adopted range, is less effective; coefficient $\Lambda_{11}$ demonstrates that $\alpha_{1}$ is the control variable with major interaction with $\sigma_{y}$.

These considerations are clearly explained by graphical plots of the models related to the mean and the variance of the quality response. They have been found according to 
TABLE 5: Central composite design and related springback quality response $(Q)$.

\begin{tabular}{|c|c|c|c|c|c|c|c|}
\hline & Run & $\sigma_{y}$ & $t$ & $\alpha_{1}$ & $\alpha_{7}$ & $\alpha_{8}$ & $Q(m m)$ \\
\hline Baseline run (target conditions) & 1 & 0 & 0 & 0 & 0 & 0 & 0.10 \\
\hline \multirow{32}{*}{ Two-level full factorial design } & 2 & 1 & 1 & 1 & 1 & 1 & 1.36 \\
\hline & 3 & -1 & 1 & 1 & 1 & 1 & 0.58 \\
\hline & 4 & 1 & -1 & 1 & 1 & 1 & 1.34 \\
\hline & 5 & -1 & -1 & 1 & 1 & 1 & 0.52 \\
\hline & 6 & 1 & 1 & -1 & 1 & 1 & 0.41 \\
\hline & 7 & -1 & 1 & -1 & 1 & 1 & 0.64 \\
\hline & 8 & 1 & -1 & -1 & 1 & 1 & 0.43 \\
\hline & 9 & -1 & -1 & -1 & 1 & 1 & 0.57 \\
\hline & 10 & 1 & 1 & 1 & -1 & 1 & 1.33 \\
\hline & 11 & -1 & 1 & 1 & -1 & 1 & 1.44 \\
\hline & 12 & 1 & -1 & 1 & -1 & 1 & 1.18 \\
\hline & 13 & -1 & -1 & 1 & -1 & 1 & 1.45 \\
\hline & 14 & 1 & 1 & -1 & -1 & 1 & 1.21 \\
\hline & 15 & -1 & 1 & -1 & -1 & 1 & 0.98 \\
\hline & 16 & 1 & -1 & -1 & -1 & 1 & 1.30 \\
\hline & 17 & -1 & -1 & -1 & -1 & 1 & 1.04 \\
\hline & 18 & 1 & 1 & 1 & 1 & -1 & 1.14 \\
\hline & 19 & -1 & 1 & 1 & 1 & -1 & 1.51 \\
\hline & 20 & 1 & -1 & 1 & 1 & -1 & 1.46 \\
\hline & 21 & -1 & -1 & 1 & 1 & -1 & 1.49 \\
\hline & 22 & 1 & 1 & -1 & 1 & -1 & 1.76 \\
\hline & 23 & -1 & 1 & -1 & 1 & -1 & 1.73 \\
\hline & 24 & 1 & -1 & -1 & 1 & -1 & 1.61 \\
\hline & 25 & -1 & -1 & -1 & 1 & -1 & 1.67 \\
\hline & 26 & 1 & 1 & 1 & -1 & -1 & 0.64 \\
\hline & 27 & -1 & 1 & 1 & -1 & -1 & 0.62 \\
\hline & 28 & 1 & -1 & 1 & -1 & -1 & 0.63 \\
\hline & 29 & -1 & -1 & 1 & -1 & -1 & 0.51 \\
\hline & 30 & 1 & 1 & -1 & -1 & -1 & 0.73 \\
\hline & 31 & -1 & 1 & -1 & -1 & -1 & 0.79 \\
\hline & 32 & 1 & -1 & -1 & -1 & -1 & 0.76 \\
\hline & 33 & -1 & -1 & -1 & -1 & -1 & 0.81 \\
\hline \multirow{10}{*}{ Axial runs } & 34 & $\mathrm{~s}$ & 0 & 0 & 0 & 0 & 1.06 \\
\hline & 35 & 0 & $\mathrm{~s}$ & 0 & 0 & 0 & 0.11 \\
\hline & 36 & 0 & 0 & $s$ & 0 & 0 & 1.80 \\
\hline & 37 & 0 & 0 & 0 & $\mathrm{~s}$ & 0 & 1.06 \\
\hline & 38 & 0 & 0 & 0 & 0 & $\mathrm{~s}$ & 1.03 \\
\hline & 39 & $-s$ & 0 & 0 & 0 & 0 & 1.03 \\
\hline & 40 & 0 & $-s$ & 0 & 0 & 0 & 0.13 \\
\hline & 41 & 0 & 0 & $-s$ & 0 & 0 & 0.85 \\
\hline & 42 & 0 & 0 & 0 & $-s$ & 0 & 1.26 \\
\hline & 43 & 0 & 0 & 0 & 0 & $-s$ & 1.97 \\
\hline \multirow{4}{*}{ Central point with noise } & 44 & 1 & 1 & 0 & 0 & 0 & 0.45 \\
\hline & 45 & -1 & 1 & 0 & 0 & 0 & 0.45 \\
\hline & 46 & 1 & -1 & 0 & 0 & 0 & 0.43 \\
\hline & 47 & -1 & -1 & 0 & 0 & 0 & 0.44 \\
\hline
\end{tabular}


TABLE 6: Regression coefficients of the response surface related to Q.

\begin{tabular}{|c|c|c|c|c|c|}
\hline \multicolumn{2}{|c|}{ Main effects of control variables } & \multicolumn{2}{|c|}{ Effects of noise variables } & \multicolumn{2}{|c|}{ Interactions control-noise Variables } \\
\hline$\gamma_{0}$ & 0.530 & $\delta_{1}$ & 0.043 & $\Lambda_{11}$ & 0.093 \\
\hline$\gamma_{1}$ & 0.151 & $\delta_{2}$ & -0.002 & $\Lambda_{12}$ & 0.004 \\
\hline$\gamma_{2}$ & 0.099 & & & $\Lambda_{13}$ & 0.001 \\
\hline$\gamma_{3}$ & 0.126 & & & $\Lambda_{21}$ & -0.007 \\
\hline$\gamma_{4}$ & 0.015 & & & $\Lambda_{22}$ & -0.008 \\
\hline$\gamma_{5}$ & 0.226 & & & $\Lambda_{23}$ & 0.003 \\
\hline$\gamma_{6}$ & -0.036 & & & & \\
\hline$\gamma_{7}$ & 0.013 & & & & \\
\hline$\gamma_{8}$ & 0.140 & & & & \\
\hline$\underline{\gamma_{9}}$ & -0.343 & & & & \\
\hline
\end{tabular}

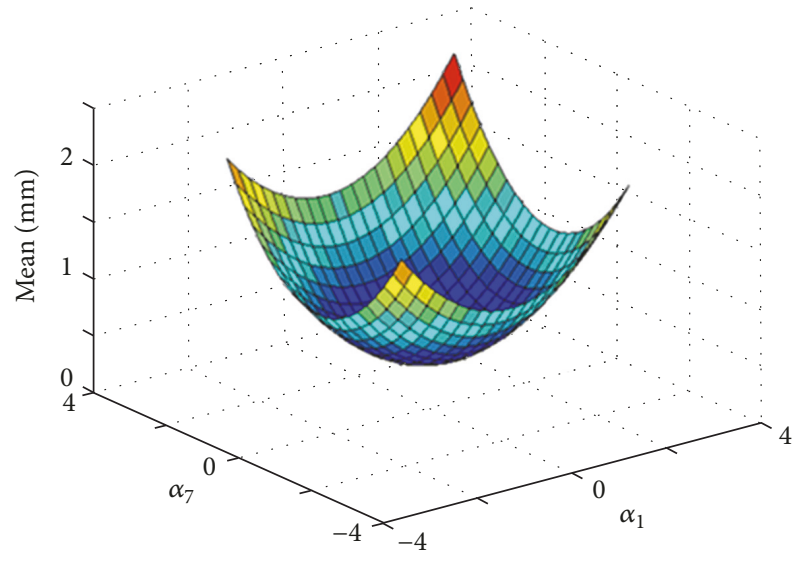

Figure 7: Model of the mean of $Q$ as a function of $\alpha_{1}$ and $\alpha_{7}\left(\alpha_{8}\right.$ coded level $=0)$. Plot is referred to the range $(-2,2)$ for both $\alpha_{1}$ and $\alpha_{7}$.

Equations (9) and (10), where the variance-covariance matrix, $\Phi$, has been defined under the hypothesis of having a $99.7 \%$ probability of material properties in the assigned tolerance, assumption that leads to a diagonal matrix with value equal to $1 / 9$. Concerning the mean model, Figure 7 clearly shows the parabolic behavior of $\alpha_{1}$ and $\alpha_{7}$, assuming $\alpha_{8}$ at the central point level.

Figures 8 and 9 allow to understand the interactions $\alpha_{1}$ $\alpha_{8}$ and $\alpha_{7}-\alpha_{8}$, respectively. They appear nonlinear and with increasing interaction moving away from the central point in both directions.

The variance of the quality response, which has been found according to Equation (10), reveals a significant quadratic effect of $\alpha_{1}$ that is able to reduce noise effect, as shown in Figure 10. It plots the effect of $\alpha_{1}$ on the standard deviation, changing the other control variables on equal levels, as shown in the legend. In this case, a minimum value exists, at $\alpha_{1}$, nearby the coded level of -0.5 . This condition differs from that one of the compensated die, named as target solution and shown in Figure 10 as a red point.

Figure 11 shows the interaction effects among control factors in terms of parametric curves. In particular, $\alpha_{8}$ is not significant while $\alpha_{7}$, suitably coupled with $\alpha_{1}$, may minimize

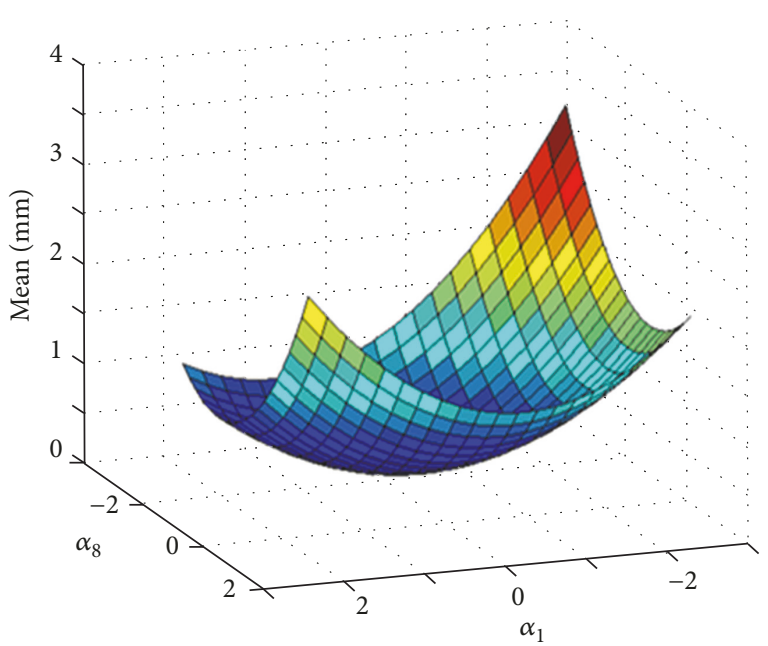

FIGURE 8: Model of the mean of $Q$ as a function of $\alpha_{1}$ and $\alpha_{8}\left(\alpha_{7}\right.$ coded level $=0)$. Plot is referred to the range $(-2,2)$ for both $\alpha_{1}$ and $\alpha_{8}$.

standard deviation. In fact, as shown in Figure 11 through the red dotted lines that represent the lower value for $\alpha_{7}$, the solution with $\alpha_{1}=1$ and $\alpha_{7}=1$ is worse than $\alpha_{1}=1$ and $\alpha_{7}=-1$ while, in case of $\alpha_{1}=-1$, the trend is reversed and the standard deviation is improved. Assuming $\alpha_{1}$ at the center point, with $\alpha_{7}=-1$, the minimum standard deviation can be achieved.

\section{Discussion}

From the analysis of the standard deviation, it can be evinced that the optimal die compensation not necessarily represents a robust solution, according to the Robust Design meaning. Through the modal shapes, assumed as control factors, it is possible to understand how changes of the compensated die may reduce noise variables effects.

In fact, combining mean and variance in a MSE function, an analytical evaluation of the Robust Design condition may be found. By the function fmincon implemented in Matlab, the robust compensation is found, $[-599,-7.21,0]$. Figure 12 shows a comparison between this "robust" die (green mesh) and the initial compensated configuration (red one). Shape 


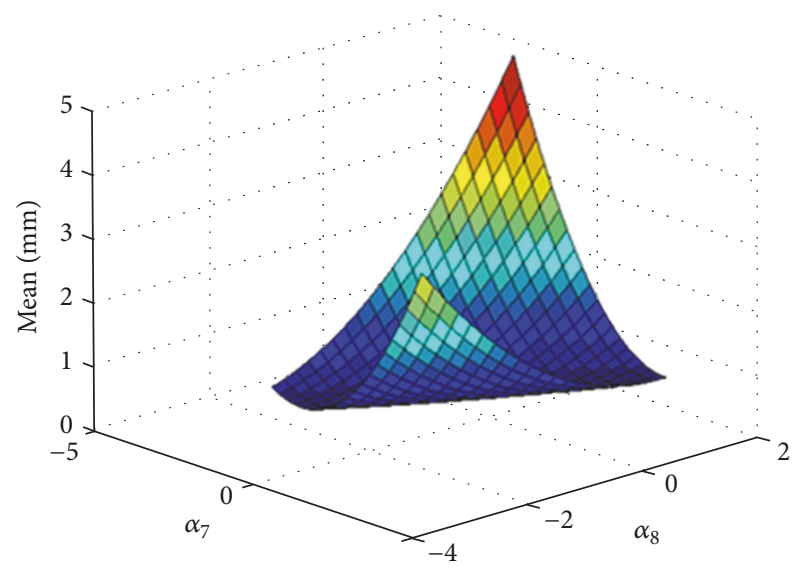

FIGURE 9: Model of the mean of $Q$ as function of $\alpha_{7}$ and $\alpha_{8}\left(\alpha_{1}\right.$ coded level $=0)$. Plot is referred to the range $(-2,2)$ for both $\alpha_{7}$ and $\alpha_{8}$.

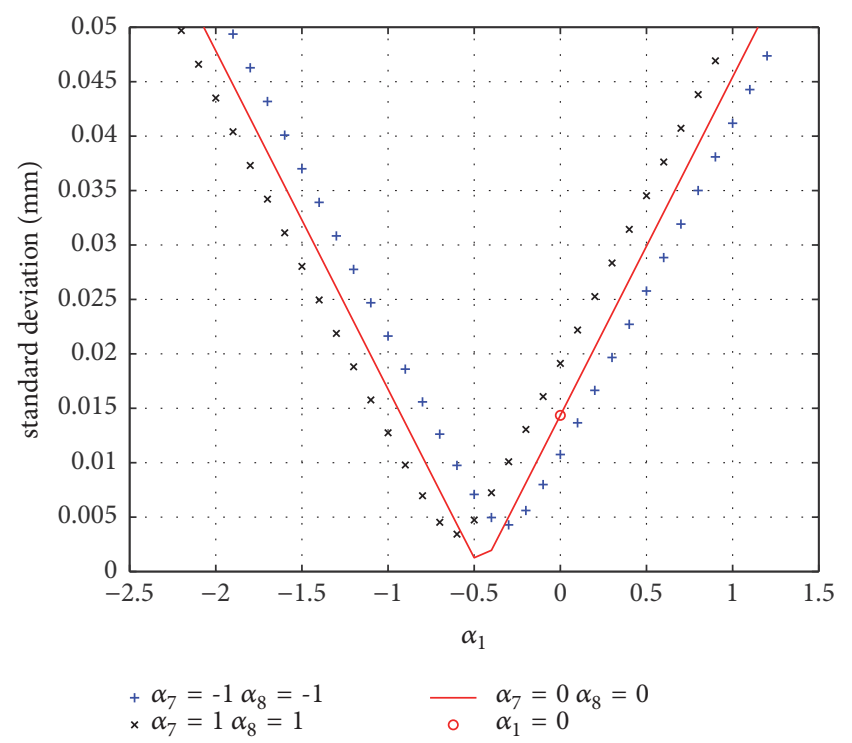

FIGURE 10: Model of the standard deviation of $Q$ as a function of $\alpha_{1}$ according to variations of the other variables on the same coded level. Standard deviation of the compensated die in red.

variations are mainly concentrated on the right flange and on the bottom of the die. No undercuts are present and shape continuity is preserved. Finally, confirmation runs with planned change of the noise variables confirm that the variance of the springback quality response is less than the variance of the target configuration associated to the initial die compensation.

\section{Conclusion}

In this paper, the die compensation principle has been extended to Robust Design applications looking for optimal die modifications that are able to reduce springback scattering of a compensated die, due to blank's scattering. It has been possible thanks to the die compensation procedure known as Shape Function optimization. By means of a suitable basis

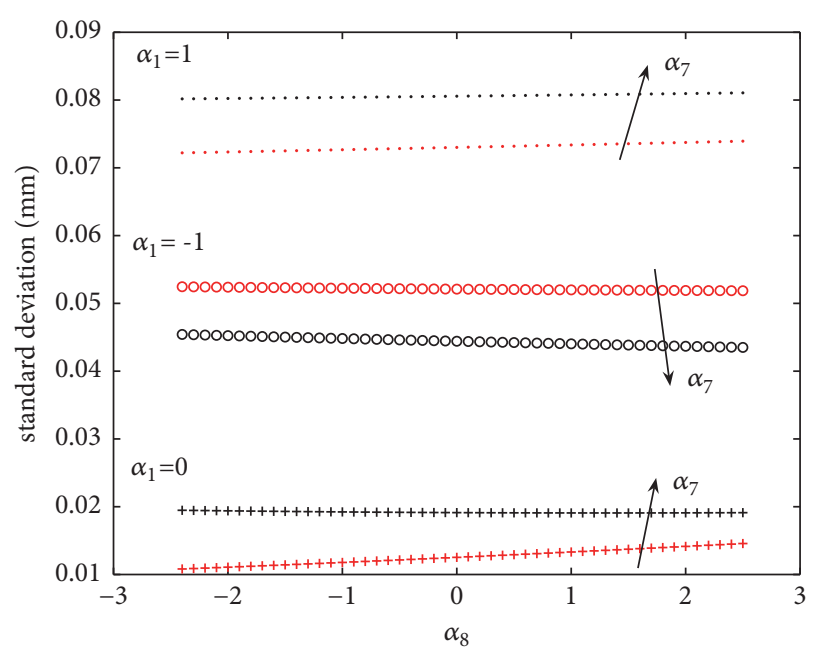

FIGURE 11: Parametric curves of the standard deviation of springback quality response, $Q$.

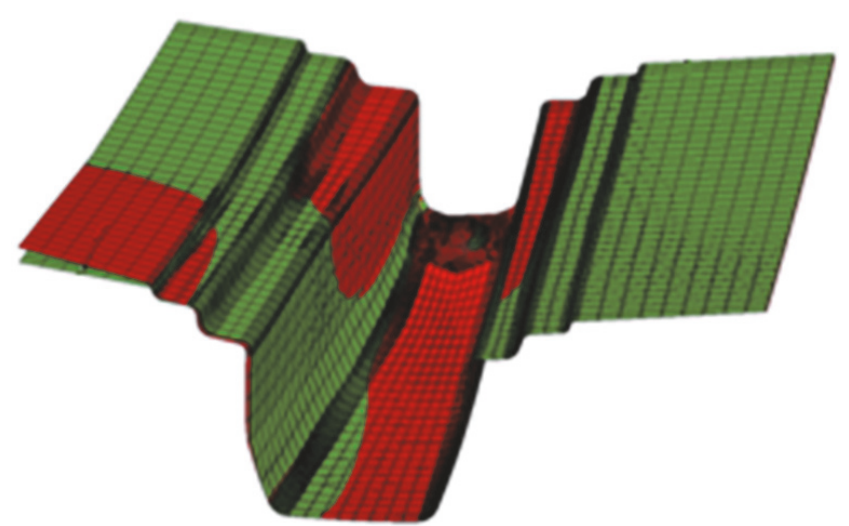

FIGURE 12: Comparison between Robust die (in green) and Compensated die (in red).

of global shapes (the shape functions derived by modal analysis), this method allows to describe geometrical variations of the tools according to global variables, the modal weights associated to each shape function, that may easily describe the correlation between die modification and process variables, according to common Robust Design procedures.

The applicability of the proposed method has been demonstrated by means of a test case, formed using an AHSS blank (referable to a Dual Phase with nominal yield stress at $1200 \mathrm{MPa}$ ) with a nominal thickness of $1.5 \mathrm{~mm}$. The preliminary die compensation, found applying the Shape Function optimization, is able to reduce springback up to $45 \%$ by means of optimal values of 10 shape functions. A preliminary validation test in the press-shop agrees with the numerical solution highlighting the same kind of corrective actions, on the same critical areas.

Dual Response Surface has been then applied to this compensated die find robust conditions for springback scattering due to initial yield stress and blank thickness (noise variables). As control variables, three global shape modifications 
have been adopted, according to the most significant shape functions found during the preliminary die compensation. Adopting noise scatter of $6 \%$, a more robust configuration for the die design has been found. The fitted model for the mean response demonstrated a nonlinear behavior of the three shape functions chosen as control variables, with some important interactions. The preliminary compensation represents a point inside the optimal area although it does not agree neither with the global minimum of the mean model nor with that of the variance. Analyzing both functions and minimizing the related MSE, an optimal compromise can be found according to small variations of the control factors.

Despite the adoption of a limited set of variables, the application demonstrates that the Dual Response Surface, if combined with the Shape Function optimization, may be easily applied to understand the effects of geometrical variations, reducing the efforts related to surface remodeling and CAD interlacing. This is mainly due to the fact that each shape function represents a global shape variation of the mesh model and it may guarantee by itself the class of the resulting die surface. In particular, for the presented test case, the first shape function represents the most effective variable for the MSE together with its interaction with the seventh ones. Both of them are related to flange distortion and vertical walls nearby the edge of the components, where major springback occurs due to the opening of the bendings.

\section{Data Availability}

All the data used to support the findings of this study are available from the authors upon request.

\section{Conflicts of Interest}

The authors declare that they have no conflicts of interest.

\section{Acknowledgments}

This work has been carried out within the Research Program of the Research Fund for Coal and Steel, Contract Number: RFCS-CT-2008-00029, entitled "An Efficient and Effective Methodology and Simulation Tools for Die Design and Springback Compensation for HSS and UHSS, "SPRINCOM"." The authors wish to thank all the partners involved in the project.

\section{References}

[1] Z. Tekiner, "An experimental study on the examination of springback of sheet metals with several thicknesses and properties in bending dies," Journal of Materials Processing Technology, vol. 145, no. 1, pp. 109-117, 2004.

[2] A. I. Burchitz, Improvement of Springback Prediction in Sheet Metal Forming. [Dissertation, thesis], University of Twente, 2008.

[3] R. Teti, S. Al Azraq, and J. Costa, "Springback prediction with FEM analysis of advanced high strength steel stamping process," IPROMS, pp. 3-14, 2006.
[4] T. Uemori, T. Okada, and F. Yoshida, "FE analysis of springback in hat-bending with consideration of initial anisotropy and the Bauschinger effect," Key Engineering Materials, vol. 177-180, pp. 497-502, 2000.

[5] F. Campana, L. Cortese, and F. Placidi, "FEM evaluation of springback after sheet metal forming: application to high strength steels of a combined isotropic-kinematic hardening model," in Proceedings of the 1st International Conference on Super High Strength Steels, p. 2, Rome, Italy, 2005.

[6] F. Campana, L. Cortese, and F. Placidi, "Finite Element Analysis of high strength steel stamping process adopting a combined kinematic-isotropic hardening model: experimental investigation on the improvements achieved in spring-back prevision," in Proceedings of IDDRG'06, pp. 19-21, Porto, Portugal, 2006.

[7] A. P. Karafillis and M. C. Boyce, "Tooling and binder design for sheet metal forming processes compensating springback error," The International Journal of Machine Tools and Manufacture, vol. 36, no. 4, pp. 503-526, 1996.

[8] R. H. Wagoner, W. Gan, K. Mao, S. Price, and F. Rasouli, "Design of sheet forming dies for springback compensation," in Proceedings of The 6th international ESAFORM, Salerno, Italy, 2003.

[9] R. A. Lingbeek, W. Gan, R. H. Wagoner, T. Meinders, and J. Weiher, "Theoretical verification of the displacement adjustment and springforward algorithms for springback compensation," International Journal of Material Forming, vol. 1, no. 3, pp. 159168, 2008.

[10] R. Wagoner, "Design of sheet forming dies for springback compensation," in Proceedings of ESAFORM, 2003, V. Brucato, Ed., pp. 7-14, Salerno, Italy, 2003.

[11] W. Gan and R. H. Wagoner, "Die design method for sheet springback," International Journal of Mechanical Sciences, vol. 46, no. 7, pp. 1097-1113, 2004.

[12] J. Weiher, B. ietman, K. Kose et al., "Controlling springback with compensation strategies," in Proceedings of the NUMIFORM2004, pp. 1011-1015, Columbus, Ohio, USA.

[13] R. Lingbeek, J. Huétink, S. Ohnimus, M. Petzoldt, and J. Weiher, "The development of finite element based springback compensation tool for sheet metal products," Journal of Materials Processing Technology, vol. 169, no. 1, pp. 115-125, 2005.

[14] R. A. Lingbeek, Aspects of a Design Tool for Springback Compensation, 2003, http://essay.utwente.nl/58267/.

[15] F. Cimolin, R. Vadori, and C. Canuto, "Springback compensation in deep drawing applications," Meccanica, vol. 43, no. 2, pp. 101-113, 2008.

[16] F. Placidi, R. Vadori, F. Cimolin, and F. Campana, "An efficient approach to springback compensation for Ultra High Strength Steel structural components for the automotive field," in Proceedings of the International Conference on New Developments on Metallurgy and Applications of High Strength Steels, pp. 193206, Buenos Aires, Argentina, May 2008.

[17] D. Campana F; Hughes, C. Pietrosanti, and F. Placidi, "Quality improvement in sheet metal forming based on the quality functions and process optimisation concepts," in Proceeding of IV IACM Conference, Buenos Aires, Argentina, June 1998.

[18] L. Fourment, R. Ducloux, S. Marie et al., "Mono and multiobjective optimization techniques applied to a large range of industrial test cases using metamodel assisted evolutionary algorithms," in Proceedings of the 10th International Conference on Numerical Methods in Industrial Forming Processes Dedicated to Professor O. C. Zienkiewicz (1921-2009), NUMIFORM 2010, pp. 833-840, Pohang, Republic of Korea, June 2010. 
[19] V. Karthik, R. J. Comstock Jr., D. L. Hershberger, and R. H. Wagoner, "Variability of sheet formability and formability testing," Journal of Materials Processing Technology, vol. 121, no. 2-3, pp. 350-362, 2002.

[20] Y. Tang and J. Chen, "Robust design of sheet metal forming process based on adaptive importance sampling," Structural and Multidisciplinary Optimization, vol. 39, no. 531, 2009.

[21] W. Zhang and R. Shivpuri, "Probabilistic design of aluminum sheet drawing for reduced risk of wrinkling and fracture," Reliability Engineering \& System Safety, vol. 94, no. 2, pp. 152161, 2009.

[22] B. Hou, W. Wang, S. Li, Z. Lin, and Z. C. Xia, "Stochastic analysis and robust optimization for a deck lid inner panel stamping," Materials and Corrosion, vol. 31, no. 3, pp. 1191-1199, 2010.

[23] J. Shu and C. Hung, "Finite element analysis and optimization of springback reduction: The "Double-Bend" technique," The International Journal of Machine Tools and Manufacture, vol. 36, no. 4, pp. 423-434, 1996.

[24] P. Chen and M. Koç, "Simulation of springback variation in forming of advanced high strength steels," Journal of Materials Processing Technology, vol. 190, no. 1-3, pp. 189-198, 2007.

[25] L. Marretta, G. Ingarao, and R. Di Lorenzo, "Design of sheet stamping operations to control springback and thinning: A multi-objective stochastic optimization approach," International Journal of Mechanical Sciences, vol. 52, no. 7, pp. 914-927, 2010.

[26] W. Wang, B. Hou, Z. Lin, and Z. C. Xia, "An engineering approach to improve the stamping robustness of high strength steels," Journal of Manufacturing Science and Engineering, vol. 131, no. 6, p. 064501, 2009.

[27] M. Gösling, H. Kracker, A. Brosius, S. Kuhnt, and A. E. Tekkaya, "Strategies for springback compensation regarding process robustness," Production Engineering Research and Development, vol. 5, no. 1, pp. 49-57, 2011.

[28] H. Wang, F. Ye, L. Chen, and E. Li, "Sheet metal forming optimization by using surrogate modeling techniques," Chinese Journal of Mechanical Engineering, vol. 30, no. 1, pp. 22-36, 2017.

[29] G. B. Broggiato, F. Campana, and E. Mancini, "Computer-aided engineering for sheet metal forming: definition of a springback quality function," Engineering with Computers, vol. 29, no. 3, pp. 319-327, 2013.

[30] J. I. Smith, M. Cardew-Hall, V. Pantano, and P. D. Hodgson, "Design, implementation and use of a knowledge acquisition tool for sheet metal forming," in Proceedings of the 2004 ASME Design Engineering Technical Conferences and Computers and Information in Engineering Conference, pp. 11-18, USA, October 2004.

[31] T. Meinders, I. A. Burchitz, M. H. A. Bonte, and R. A. Lingbeek, "Numerical product design: springback prediction, compensation and optimization," The International Journal of Machine Tools and Manufacture, vol. 48, no. 5, pp. 499-514, 2008.

[32] K. M. Liew, T. Ray, H. Tan, and M. J. Tan, "Evolutionary optimization and use of neural network for optimum stamping process design for minimum springback," Journal of Computing and Information Science in Engineering, vol. 2, no. 1, pp. 38-44, 2002.

[33] D. J. Ewins, Modal Testing: Theory, Practice and Application. Research Studies Pre, 2nd edition, 2000.

[34] J. L. Chaboche, "Constitutive equations for cyclic plasticity and cyclic viscoplasticity," International Journal of Plasticity, vol. 5, no. 3, pp. 247-302, 1989.
[35] G. Taguchi, S. Chowdhury, and Y. Wu, Taguchi's Quality Engineering Handbook, John Wiley \& Sons, 2005.

[36] R. H. Myers, A. I. Khuri, and G. Vining, "Response surface alternatives to the Taguchi robust parameter design approach," The American Statistician, vol. 46, no. 2, pp. 131-139, 1992.

[37] D. C. Montgomery, Design and Analysis of Experiments, John Wiley \& Sons, Hoboken, NJ, USA, 2009.

[38] T. Jansson, L. Nilsson, and R. Moshfegh, "Reliability analysis of a sheet metal forming process using Monte Carlo analysis and metamodels," Journal of Materials Processing Technology, vol. 202, no. 1-3, pp. 255-268, 2008.

[39] Midterm, "An Efficient and effective methodology and simulation tools for die design and springback compensation for HSS and UHSS, (SPRINCOM)," Midterm Report TGS7.02/08, Financing by the Research Program of the Research Fund for Coal and Steel, contract no. RFSR-CT-2008-00029, 2008. 


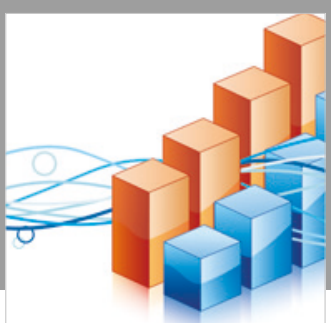

Advances in

Operations Research

\section{-n-m}
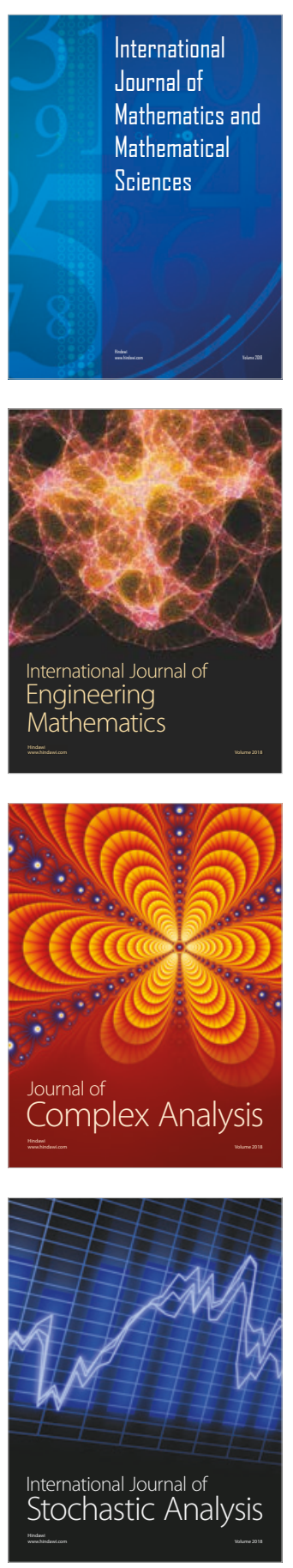
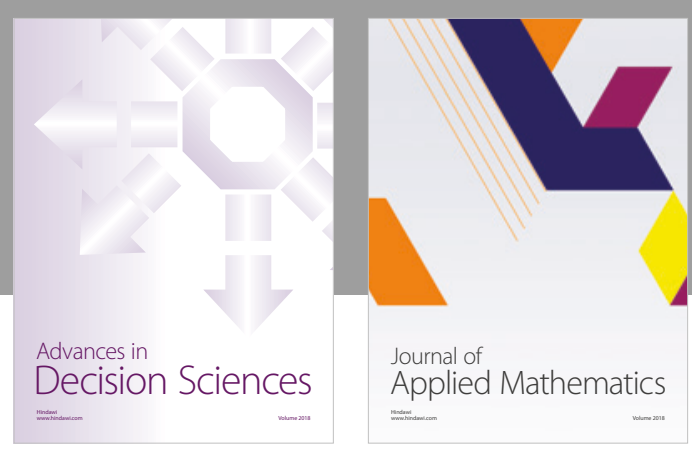

Journal of

Applied Mathematics
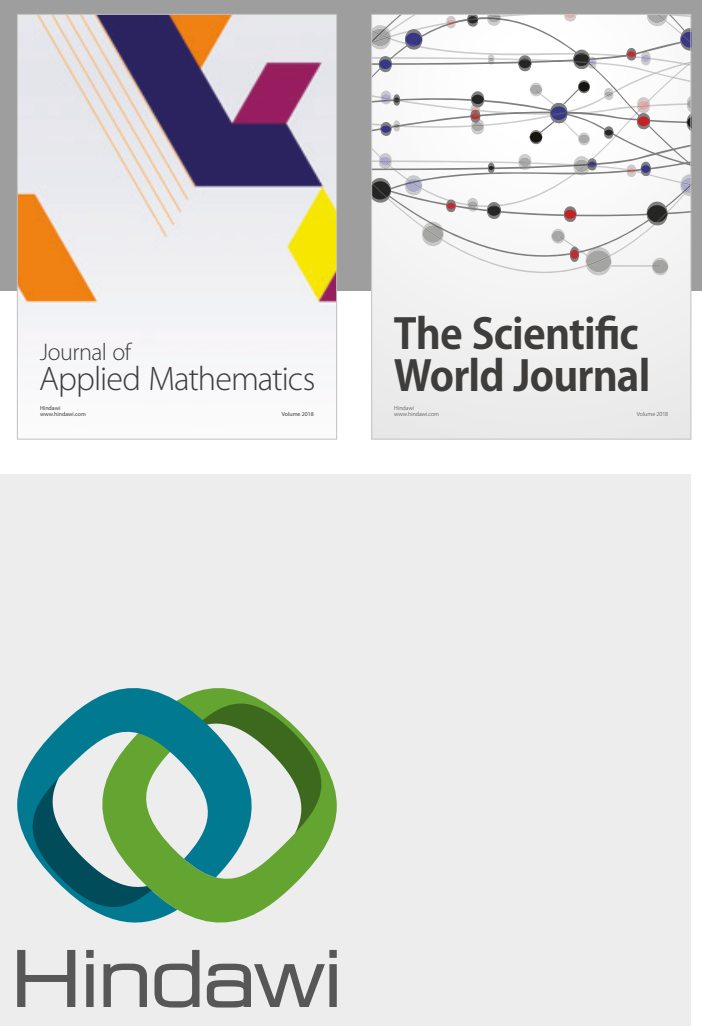

Submit your manuscripts at

www.hindawi.com

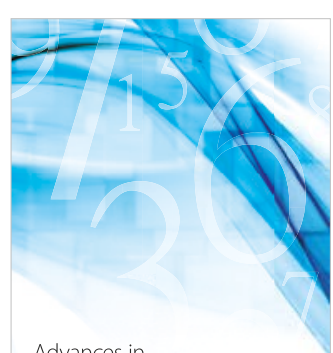

Advances in
Numerical Analysis
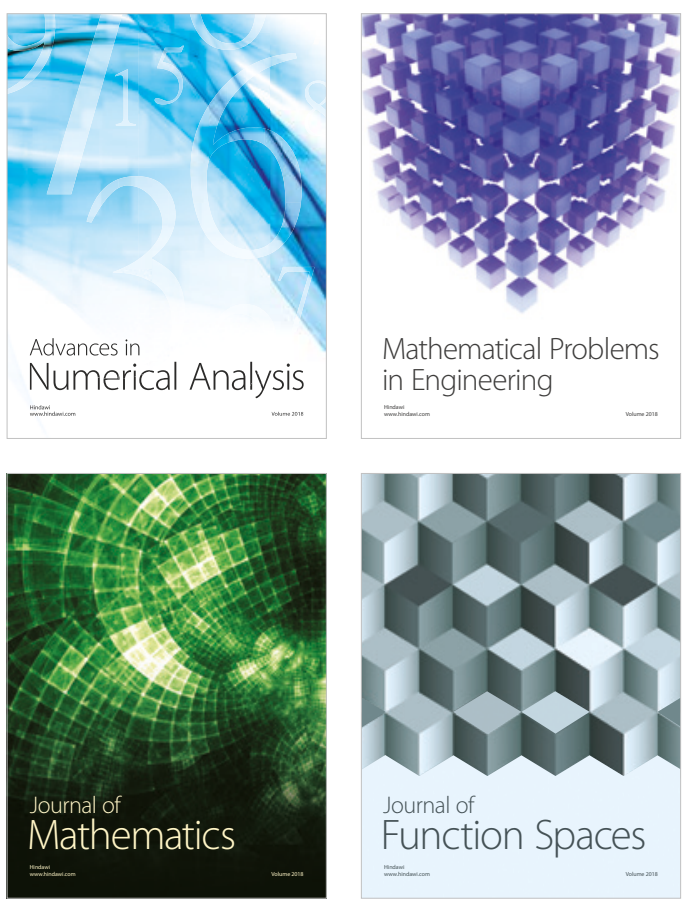

Mathematical Problems in Engineering

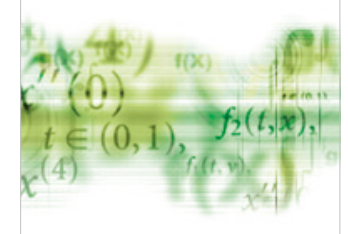

International Journal of

Differential Equations

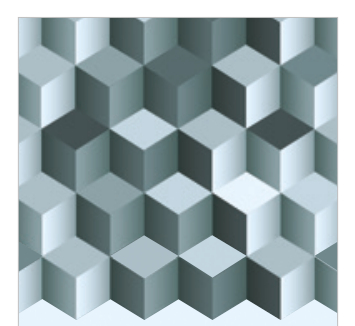

Journal of

Function Spaces

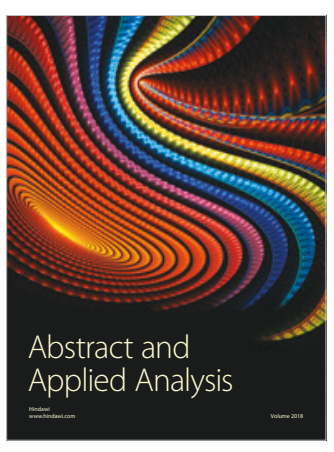

The Scientific

World Journal

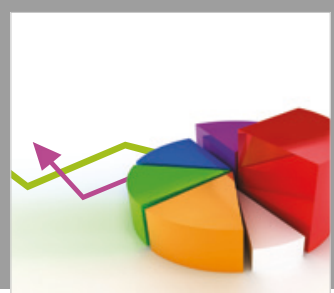

Journal of

Probability and Statistics
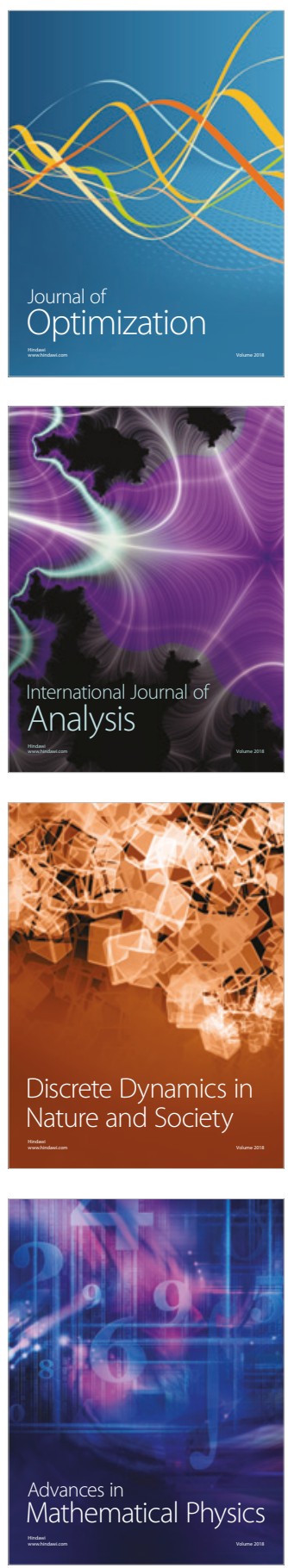